\title{
ON $n$-METACALORIC FUNCTIONS
}

\section{AVNER FRIEDMAN}

Introduction. Some theorems on polyharmonic functions can be generalized to $n$-metaharmonic functions, that is functions $u(x)$ (x $\left.=\left(x_{1}, \cdots, x_{N}\right)\right)$ satisfying equations of the form $p(\Delta) u(x)=0$, where $p(t)$ is a polynomial in $t$ of degree $n$ and $\Delta=\sum \partial^{2} / \partial x_{i}^{2}$. We especially refer to [2] and [4].

In a recent paper, [5], Nicolesco has developed the theory of $n$ caloric functions, that is functions satisfying the parabolic equation $\Omega^{n} u(x, t)=0$, where $\Omega u=\Delta u-\partial / \partial t$.

The aim of this paper is to generalize some of the theorems on $n$-caloric functions to $n$-metacaloric functions, that is to functions satisfying $p(\Omega) u(x, t)=0$. Our results are analogous to the results of Ghermanesco [4] and the author [2], in the theory of $p(\Delta) u(x)=0$. For the sake of simplicity we take $N=1, \Delta=\partial^{2} / \partial x^{2}$, but all the results hold for the general case $\Delta=\sum \partial^{2} / \partial x_{i}^{2}$.

By a solution of $p(\Omega) u(x, t)=0$, we shall always mean a function which possesses all the derivatives which appear in $p(\Omega)$ and which satisfies $p(\Omega) u(x, t)=0$.

1. Theorem 1. Let $p(z)$ be the polynomial $\prod_{i=1}^{k}\left(z-\alpha_{i}\right)^{n_{i}}$ and consider the equation

$$
p(\Omega) u(x, t)=0
$$

in a domain $D$. Then the general solution of (1) has the form

$$
u(x, t)=\sum_{i=1}^{k} \sum_{j=0}^{n_{i}-1} t^{j} u_{j}^{\alpha_{i}}(x, t),
$$

where $u_{j}^{\alpha_{i}}(x, t)$ are solutions in $D$ of $\left(\Omega-\alpha_{i}\right) u(x, t)=0$.

Proof. It can easily be proved by induction that if $v(x, t)$ satisfies $(\Omega-\alpha) v(x, t)=0$, then

$$
(\Omega-\alpha)^{k}\left(t^{k} v(x, t)\right)=(-1)^{k} k ! v(x, t) .
$$

From this remark it follows that the $u$ given by (2) satisfies (1). To prove the converse, consider first the case of $p(\Omega)=(\Omega-\alpha)^{n}$. The proof is by induction on $n$. It is sufficient to show that if $u$ satisfies $(\Omega-\alpha)^{n}$

Received by the editors April 2, 1956 and, in revised form, September 4, 1956. 
$u=0$, then there exists a function $v(x, t)$ satisfying

$$
(\Omega-\alpha) v=0, \quad(\Omega-\alpha)^{n-1}\left(t^{n-1} v\right)=(\Omega-\alpha)^{n-1} u .
$$

For then we write $u=t^{n-1} v+\left(u-t^{n-1} v\right)$ and use the inductive assumption. By the remark made at the beginning of the proof it is obvious that the function

$$
v(x, t)=\frac{(-1)^{n-1}}{(n-1) !}(\Omega-\alpha)^{n-1} u(x, t)
$$

satisfies (3).

Having proved the theorem for $p(\Omega)=(\Omega-\alpha)^{n}$, we can prove the general case of $p(\Omega)=\prod_{i=1}^{k}\left(\Omega-\alpha_{i}\right)^{n_{i}}$ by induction on $k$, in exactly the same way as in the case of $p(\Delta)$ (see [2]).

REMARK. That the representation (2) is unique, is easily seen by applying $\prod_{i=1 ; i \neq j}^{k}\left(\Omega-\alpha_{i}\right)^{n_{i}}\left(\Omega-\alpha_{j}\right)^{m} m=n_{j}-1, \cdots, 1,0$ to both sides of (2).

Corollary. The solutions $u(x, t)$ of $p(\Omega) u=0$ have the following properties:

(a) $u(x, t)$ is infinitely differentiable in $(x, t)$,

(b) for every $t_{0}, u\left(x, t_{0}\right)$ is analytic in $x$, and

(c) for every $x_{0}, u\left(x_{0}, t\right)$ belongs to the second class of Holmgren (see $[3])$.

The corollary follows from Theorem 1 and the fact that metacaloric functions possess the properties (a), (b), (c).

2. Lemma 1. Let $u(x, t)$ be a solution of $(\Omega-\alpha) u(x, t)=0$ in the strip $t_{1} \leqq t \leqq t_{2}$ and

$$
|u(x, t)| \leqq M e^{K x^{2}} \quad\left(t_{1} \leqq t \leqq t_{2}\right) .
$$

Then for every $\epsilon>0$, there exists a constant $M^{\prime}=M^{\prime}(\epsilon, N, M)$ such that for $t, t_{1}+\epsilon \leqq t \leqq t_{2}$,

$$
\left|\frac{\partial^{k} u(x, t)}{\partial x^{k-j} \partial t^{j}}\right| \leqq M^{\prime} e^{2 K x^{2}} \quad(0 \leqq j \leqq k, 0 \leqq k \leqq N) .
$$

Proof. It is sufficient to prove that for every $t_{0}, t_{1} \leqq t_{0}<t_{2}$ and for every $t$ which satisfies $t_{0}+\epsilon \leqq t \leqq t_{0}+1 / 8 K-\epsilon, t \leqq t_{2}$,

$$
\left|\frac{\partial^{k} u(x, t)}{\partial x^{k-j} \partial t^{j}}\right| \leqq M^{\prime} e^{2 K x^{2}} \quad(0 \leqq j \leqq k, 0 \leqq k \leqq N)
$$

where $M^{\prime}$ depends on $\epsilon, N, M$. 
Without loss of generality, we may assume $t_{0}=0$. Consider the function

$$
v(x, t)=\frac{e^{-\alpha t}}{2(\pi t)^{1 / 2}} \int_{-\infty}^{\infty} e^{-(x-\xi)^{2} / 4 t} u(\xi, 0) d \xi
$$

and apply to it the operator $\partial^{k} / \partial x^{k-j} \partial t^{i}$. Substituting $x-\xi=-2 t^{1 / 2} s$ we have

$$
\begin{aligned}
\left|\frac{\partial^{k} v(x, t)}{\partial x^{k-j} \partial t^{j}}\right| & \leqq \int_{-\infty}^{\infty}\left|\frac{\partial^{k}}{\partial x^{k-j} \partial t^{i}}\left(\frac{e^{-\alpha t} e^{-(x-\xi)^{2} / 4 t}}{2(\pi t)^{1 / 2}}\right)\right| M e^{K\left(x+2 t^{1 / 2} s\right)^{2}} d s \\
& \leqq M_{1} \int_{-\infty}^{\infty} s^{2 N} e^{-s^{2}} e^{2 K\left(x^{2}+4 t t^{2}\right)} d s \leqq M^{\prime} e^{2 K x^{2}} .
\end{aligned}
$$

It remains to prove that $v(x, t)=u(x, t)$. Since the function $w=u-v$ satisfies

$$
w(x, 0)=0, \quad(\Omega-\alpha) w(x, t)=0, \quad|w(x, t)| \leqq\left(M+M^{\prime}\right) e^{2 K x^{2}},
$$

it follows from [1] that $w(x, t) \equiv 0$.

3. THEOREM 2. Let $u(x, t)$ be a solution of $(\Omega-\alpha) u=0$ in the strip $t_{1} \leqq t \leqq t_{2}$ and $|u(x, t)| \leqq M e^{K x^{2}}\left(t_{1} \leqq t \leqq t_{2}\right)$. Then for every $h, t$ which satisfy $t_{1}<t \leqq t_{2}, t_{1}<t-h, 0<h<1 / 8 K$, the following mean-value formula holds:

$$
\mu(u ; h) \equiv \frac{1}{2(\pi h)^{1 / 2}} \int_{-\infty}^{\infty} e^{-(x-\xi)^{2} / 4 h} u(\xi, t-h) d \xi=e^{\alpha h} u(x, t) .
$$

Proof. Let $\phi(h)=2(\pi h)^{1 / 2} \mu(u ; h)$. Using Lemma 1 and integrating by parts, we have

$$
\frac{\partial \phi}{\partial h}=\int_{-\infty}^{\infty} e^{-(x-\xi)^{2} / 4 h}\left[\Omega(u \xi, t-h)+\frac{1}{2 h} u(\xi, t-h)\right] d \xi=\left(\alpha+\frac{1}{2 h}\right) \phi,
$$

or $\phi(h)=c h^{1 / 2} e^{\alpha h}$. To find $c$, observe that $\mu(u ; h)=u(x, t)+o(1)$ $(h \rightarrow 0)$, so that $c=2(\pi)^{1 / 2} u(x, t)$ and we have proved (5).

4. Definition. A function $u(x, t)$ defined in a strip $t_{1} \leqq t \leqq t_{2}$, is said to be of type $(K, n)$ (we suppose $K \neq 0$ ), if $\Omega^{k} u(x, t)$ $(k=0,1, \cdots, n)$ exist and satisfy

$$
\left|\Omega^{k} u(x, t)\right| \leqq M e^{K x^{2}} \quad\left(t_{1} \leqq t \leqq t_{2}, k=0,1, \cdots, n\right),
$$

and of type $(0, n)$, if for every positive $\epsilon$ it is of type $(\epsilon, n)$.

THEOREM 3. Let $u(x, t)$ be a solution of $p(\Omega) u=0$, where $p(z)$ 
$=\prod_{i=1}^{k}\left(z-\alpha_{i}\right)^{n_{i}}\left(\sum n_{i}=n\right)$, and suppose $u$ to be of type $(K, n-1)$ in the strip $t_{1} \leqq t \leqq t_{2}$. Then, for every $t, h$ which satisfy

$$
\begin{gathered}
t_{1}<t \leqq t_{2}, \quad t_{1}<t-h, \quad 0<h<1 / 8 K, \\
\mu(u ; h)=\sum_{i=1}^{k} \sum_{j=0}^{n_{i}-1}(t-h)^{j} e^{\alpha_{i} h} u_{j}^{\alpha_{i}}(x, t) .
\end{gathered}
$$

Proof. Since $u(x, t)$ is of type $(K, n-1)$, for every polynomial $q(z)$ of degree $\leqq n-1,|q(\Omega) u(x, t)| \leqq M e^{K x^{2}}$. Choosing $q(z)$ $=\prod_{i=1 ; i \neq j}^{k}\left(z-\alpha_{i}\right)^{n_{i}}\left(z-\alpha_{j}\right)^{m}, m=n_{j}-1, n_{j}-2, \cdots, 0$ we can easily see that each $u_{j}^{\alpha t}(x, t)$ in $(2)$ is of type $(K, 0)$. Therefore, by Theorem 2

$$
\begin{aligned}
\mu(u ; h) & =\sum_{i=1}^{k} \sum_{j=0}^{n_{i}-1} \frac{1}{2(\pi h)^{1 / 2}} \int_{-\infty}^{\infty} e^{-(x-\xi)^{2} / 4 h}(t-h)^{j} u_{j}^{\alpha_{i}}(\xi, t-h) d \xi \\
& =\sum_{i=1}^{k} \sum_{j=0}^{n_{i}-1}(t-h)^{j} \mu\left(u_{j}^{\alpha_{i}} ; h\right)=\sum_{i=1}^{k} \sum_{j=0}^{n_{i}-1}(t-h)^{j} e^{\alpha_{i} h} u_{j}^{\alpha_{i}}(x, t) .
\end{aligned}
$$

5. We now prove a generalization of Liouville's Theorem:

THEOREM 4. Let $p(z)$ be a polynomial of the form $\prod_{i=1}^{k}\left(z-\alpha_{i}\right)^{n_{i}}$ $\left(\sum n_{i}=n\right)$ with $\operatorname{Re}\left\{\alpha_{i}\right\} \geqq 0$, and let $u(x, t)$ satisfy $p(\Omega) u(x, t)=0$ in the strip $-\infty<t \leqq t_{2}$. Suppose $u(x, t)$ to be of the type $(0, n-1)$ in every strip $t_{1} \leqq t \leqq t_{2}$. If $u(x, t)$ is bounded and if

$$
\lim _{h \rightarrow \infty} \mu(u ; h) \text { exists, }
$$

then $u(x, t) \equiv$ const.

Proof. Using Theorem 1 with $D:-\infty<t \leqq t_{2}$, we derive equation (6) for all positive $h$. Write (6) in the form

$$
\mu(u ; h)=\sum_{i=1}^{n} F_{i}(x, t) \phi_{i}(h) .
$$

Letting $h \rightarrow \infty$ and comparing the behaviour at infinity of the functions $\phi_{i}(h)$, we conclude that

$$
\mu(u ; h)=\sum_{\text {Rol } \left.\alpha_{i}\right)=0 ; \alpha_{i} \neq 0} e^{\alpha_{i} h} u_{0}^{\alpha_{i}}(x, t)+u_{0}^{0}(x, t) .
$$

The sum on the right-hand side in (9) is an almost periodic function in the sense of Bohr, and since its limit (as $h \rightarrow \infty$ ) exists, it must vanish. We obtain

$$
\mu(u ; h)=u_{0}^{0}(x, t) .
$$


Noting that $\mu(u ; h)=u(x, t)+o(1)(h \rightarrow 0)$, we conclude

$$
\mu(u ; h)=u(x, t) .
$$

By [5], $\mu(u ; h)=u(x, t)+h \Omega u(x, t)+o(h)(h \rightarrow 0)$ and together with (10), $\Omega u(x, t)=0$. Since for caloric functions Liouville's Theorem is true (see [5]), the proof is completed.

REMARK. From the proof it follows that in case that either $\operatorname{Re}\left\{\alpha_{i}\right\}>0$ or $\alpha_{i}=0(1 \leqq i \leqq k)$, the assumption (7) is superfluous.

6. In this section we prove that equation (8) characterizes the $n$-metacaloric functions.

Theorem 5. Let $u(x, t)$ be defined in the strip $t_{1} \leqq t \leqq t_{2}$ and possess second-order continuous derivatives which satisfy

$$
|u(x, t)|, \quad\left|\frac{\partial u(x, t)}{\partial t}\right|, \quad\left|\frac{\partial u(x, t)}{\partial x}\right|, \quad\left|\frac{\partial^{2} u(x, t)}{\partial x^{2}}\right| \leqq M e^{K x^{2}} .
$$

Then for every $t, h$ which satisfy

$$
\begin{aligned}
& t \leqq t_{2}, \quad t_{1} \leqq t-h<t_{2} \quad 0<h<\frac{1}{4 K}, \\
& \Omega \mu(u ; h)=\frac{\partial \mu(u ; h)}{\partial h} .
\end{aligned}
$$

Proof. As in the proof of Theorem 3,

$$
\frac{\partial\left(2(\pi h)^{1 / 2} \mu\right)}{\partial h}=\int_{-\infty}^{\infty} e^{-(x-\xi)^{2} / 4 h}\left(\Omega u+\frac{1}{2 h} u\right) d \xi .
$$

Since on the other hand

$$
\frac{\partial\left(2(\pi h)^{1 / 2} \mu\right)}{\partial h}=2(\pi h)^{1 / 2} \frac{\partial \mu}{\partial h}+\frac{2 \pi^{1 / 2}}{2 h^{1 / 2}} \mu,
$$

we conclude

$$
\frac{\partial \mu}{\partial h}=\frac{1}{2(\pi h)^{1 / 2}} \int_{-\infty}^{\infty} e^{-(x-\xi)^{2} / 4 h} \Omega u(\xi, t-h) d \xi=\Omega \mu .
$$

Suppose that each $\Omega^{k} u(x, t)(k=0,1, \cdots, n-1)$ satisfies the assumptions of Theorem 5 . We then have

$$
\begin{aligned}
\Omega^{k} \mu(u ; h) & =\frac{1}{2(\pi h)^{1 / 2}} \int_{-\infty}^{\infty} e^{-(x-\xi)^{2} / 4 h} \Omega^{k} u(\xi, t-h) d \xi \\
& =\Omega^{k} u(x, t)+o(1) \quad \mathrm{l}(k=0,1, \cdots, n ; h \rightarrow 0) .
\end{aligned}
$$


Suppose $u(x, t)$ satisfies (8) with $n$ times differentiable $\phi_{i}(h)$. Then by Theorem 5

$$
\Omega^{k} \mu=\sum_{i=1}^{n} F_{i}(x, t) \frac{d^{k} \phi_{i}(h)}{d h^{k}} \quad(k=0,1, \cdots, n) .
$$

Since this system has a nontrivial solution, we conclude that

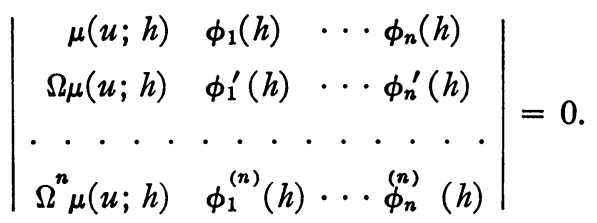

Taking $h \rightarrow 0$ and using (12) it follows that $u$ is $n$-metacaloric. Hence

Theorem 6. If $u(x, t)$ is n-metacaloric and of type $(K, n-1)$ in the strip $t_{1} \leqq t \leqq t_{2}$, then

$$
\mu(u ; h)=\sum_{i=1}^{n} F_{i}(x, t) \phi_{i}(h) \quad\left(t_{1}<t-h, t<t_{2}, 0<h<1 / 8 K\right),
$$

$\phi_{i}(h)$ are analytic in $h$ and $F_{i}(x, t)$ are indefinitely differentiable in $(x, t)$ and analytic in $x$. Conversely, if

$$
\begin{aligned}
&\left|\Omega^{k} u(x, t)\right|, \quad\left|\frac{\partial}{\partial t} \Omega^{k} u(x, t)\right|, \quad\left|\frac{\partial}{\partial x} \Omega^{k} u(x, t)\right|, \\
&\left|\frac{\partial^{2}}{\partial x^{2}} \Omega^{k} u(x, t)\right| \leqq M e^{K x^{2}} \quad(k=0, \cdots, n-1)
\end{aligned}
$$

and if (8) holds with $n$ times differentiable $\phi_{i}(h)$ and for $h$ sufficiently small, then $u(x, t)$ is $n$-metacaloric and of type $(K, n-1)$.

7. Lemma 2. Let $u(x, t)$ be $n$-metacaloric and of type $(K, n-1)$ in the strip $t_{1} \leqq t \leqq t_{2}$. Then (14) holds in any interval $t_{1}+\epsilon \leqq t \leqq t_{2}$ and with $2 K$ in place of $K$.

Proof. Write $u$ in the form (2). As in the proof of Theorem 3, each $u_{j}^{\alpha_{i}}$ is of type $(K, 0)$. Apply Lemma 1 to $u_{j}^{\alpha_{i}}$.

Suppose $u(x, t)$ to be $n$-metacaloric and of type $(K, n-1)$. Using Lemma 2, we have

$$
\Omega^{k} \mu(u ; h)=\frac{1}{2(\pi h)^{1 / 2}} \int_{-\infty}^{\infty} e^{-(x-\xi)^{2} / 4 h} \Omega^{k} u(\xi, t-h) d \xi .
$$

Since $p(\Omega) u=0$, we also have $p(\Omega) \mu(u ; h)=0$. By Theorem 5 we get

$$
p\left(\frac{\partial}{\partial h}\right) \mu(u ; h)=0 \text {. }
$$


Conversely, if $\mu(u ; h)$ satisfies (15) and $u$ satisfies (14), then by Theorem 5 and $(15)$ we get $p(\Omega) \mu(u ; h)=0$. Taking $h \rightarrow 0$, we conclude that $p(\Omega) u(x, t)=0$. We have proved:

THEOREM 7. If $u(x, t)$ satisfies $p(\Omega) u=0$ and if it is of type $(K, n-1)$, then

$$
p(\partial / \partial h) \mu(u ; h)=0 \quad\left(t_{1}<t-h<t_{2}, 0<h<1 / 8 K\right) .
$$

Conversely, from (14) and (16) follows $p(\Omega) u=0$.

8. We shall derive a special form of equation (8). From (6) it is clear that the $\phi_{i}(h)$ are independent solutions of $p(\partial / \partial h) \phi(h)=0$, therefore their Wronskian is different from zero. Applying $\Omega^{k}$ to both sides of (8) and letting $h \rightarrow 0$, we get the system

$$
\begin{aligned}
\mu(u ; h) & =\sum_{i=1}^{n} F_{i}(x, t) \phi_{i}(h), \\
\Omega^{k} u(x, t) & =\sum_{i=1}^{n} F_{i}(x, t) \frac{d^{k} \phi_{i}(0)}{d h^{k}} \quad(k=0,1, \cdots, n-1),
\end{aligned}
$$

from which it follows that

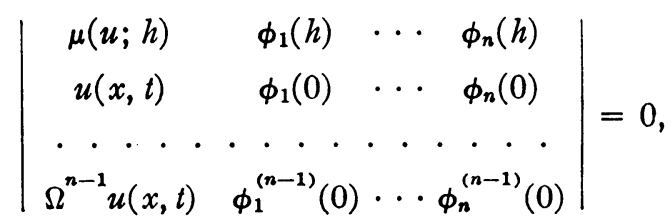

or

$$
\mu(u ; h)=\sum_{k=0}^{n-1} c_{k}(h) \Omega^{k} u(x, t),
$$

where $c_{k}(h)$ are linear combinations of expressions $h^{i} e^{\alpha i h}$.

\section{REFERENCES}

1. C. L. Eidelman, Estimates of solutions of parabolic systems and some applications, Rec. Math. (Mat. Sbornik) N.S. vol. 33 (1953) pp. 359-382.

2. A. Friedman, On n-metaharmonic functions and harmonic functions of infinite order, Proc. Amer. Math. Soc. vol. 8 (1957) pp. 223-229.

3. E. Goursat, Cours d'analyse, vol. 3, Paris, 1923.

4. M. Ghermanesco, Sur les moyennes successives des fonctions, Bull. Soc. Math. France vol. 62 (1934) pp. 245-264.

5. M. Nicolesco, L'equation itérée de la chaleur, Studii Cercetãri Math. vol. 5 (1954) pp. 243-332.

The Hebrew University, Jerusalem 